26\%; $\mathrm{p}=0.0034)$, while patients with PD were less likely to be discharged home in the group without thrombectomy $(32 \%$ vs. $51 \% ; \mathrm{p}<0.0001)$. Length of hospital stay was similar between groups (range 4.3 - 8.7 days). Inpatient mortality was significantly higher in patients with $\mathrm{AD}(5 \%$ vs. $4 \%$; $\mathrm{p}<0.0001)$ and PD $(5 \%$ vs. $4 \% ; \mathrm{p}=0.044)$ in the group that did not undergo MT.

Conclusions Patients with co-existing neurological disorders underwent mechanical thrombectomy at a lower rate compared to other patients. Further studies should elucidate factors affecting the rate MT in the United States.

Disclosures L. Brasiliense: None. H. Abdulrazek: None. P. Aguilar-Salinas: None. H. Abbad: None. T. Dumont: None. M. El-Ghanem: None.

\section{E-090 NOVEL ASPIRATION CATHETER WITH HYDRO- SEPARATOR TECHNOLOGY FOR TOUGH CLOTS}

${ }^{1} \mathrm{~N}$ Kaneko*, 'L Guo, ${ }^{2} \mathrm{Y}$ Komuro, ${ }^{1} \mathrm{~S}$ Tateshima. 'Department of Radiological Sciences, UCLA, Los Angeles, CA; ${ }^{2}$ Department of Neurology, UCLA, Los Angeles, CA

\subsection{6/neurintsurg-2020-SNIS.123}

Background Large clot burden and fibrin clots remain challenging for mechanical thrombectomy. The aim of this study is to assess a new-generation aspiration system with hydro-separator technology that works regardless of clot size or composition.

Materials and Methods The Neurostar thrombectomy system is comprised of a $6 \mathrm{~F}$ Neurostar catheter and a Saline Drive Unit (SDU) with a peristaltic pump, which creates a stream of saline on the tip of the catheter to macerate the thrombus during aspiration (hydro-separator). The Neurostar catheter as well as two commercially available thrombectomy devices, the Solitaire FR stent retriever and Sofia Plus aspiration catheter, were tested to compare single-pass recanalization performance in challenging situations. Fibrous clots and cohesive erythrocyte-rich clots were produced from porcine blood. A segment of fibrous clot was placed in the MCA distal to M1 and proximal to $\mathrm{M} 2$ in a tortuous in-vitro intracranial vascular model, or a relatively long $(20 \mathrm{~mm})$ erythrocyte-rich clot was placed in the middle segment of the M1. Mechanical thrombectomy using each device was repeated 10 times with fibrous clots and erythrocyte-rich clots. Success recanalization was defined as clot removal without visible fragmentation or migration.

Results The Neurostar catheter led to significantly better single-pass recanalization performance $(18 / 20)$ compared to the Sofia Plus aspiration catheter (11/20) and Solitaire FR stent retriever (10/20). All of the recanalization procedures with the Sofia Plus required complete removal of the catheter because the clot was stuck at the tip or could not be ingested entirely. In contrast, the Neurostar catheter could ingest the clot and remain at the site of occlusion in all of the successful single pass recanalization procedures.

Conclusions Mechanical thrombectomy with the new aspiration system with hydro-separator resulted in higher success rates than a commercially available stent retriever and aspiration catheter in this experimental model. Further in vivo studies should be performed to confirm its performance.

Disclosures N. Kaneko: 2; C; Walk Vascular. L. Guo: None. Y. Komuro: None. S. Tateshima: 2; C; Walk Vascular, Medtronic.

\section{E-091 HIGH INFARCT GROWTH RATE IS ASSOCIATED WITH POOR FUNCTIONAL OUTCOME IN PATIENTS WITH ACUTE LARGE VESSEL OCCLUSION AND SUCCESSFUL REVASCULARIZATION}

D Quispe Orozco, J Sequeiros Chirinos, C Zevallos Mau, A Mendez Ruiz, S Abdelkarim, S Ansari, A Mendez Ruiz, M Farooqui, S Dandapat, S Ortega Gutierrez*. University of lowa Hospitals and Clinics, lowa City, IA

\subsection{6/neurintsurg-2020-SNIS.124}

Introduction Despite successful revascularization (TICI $2 \mathrm{~b}$ or 3), almost half of the acute ischemic stroke patients with large vessel occlusion have a poor outcome. We aim to evaluate the extent by which collateral compensation is independently associated with functional outcome after successful recanalization. Methods We retrospectively reviewed all acute ischemic stroke (AIS) patients with anterior large vessel occlusion (LVO) who underwent mechanical thrombectomy with successful revascularization in our comprehensive stroke center from 2014 to 2019. Inclusion criteria were age $>18$, time from last known well to reperfusion $<24$ hours, and patients who underwent CTP or MRI before and MRI after thrombectomy (within 24 hours). Ischemic core volume in CTP was measured as the relative $\mathrm{CBF}<30 \%$ volume of that normal tissue. MRI ischemic core volumes were calculated manually and by using Automatic Rapid Software (subtraction of 620 ACD volume$\mathrm{CBF}<30 \%$ in the ischemic hemisphere). Infarct growth rate was defined as: (infarct volume post recanalization - infarct volume before recanalization)/(time from CTP or MRI before recanalization to MRI after recanalization). Functional outcome was measured by the modified Rankin Score (mRS) at 3 months dichotomized as good $(\mathrm{mRS} \leq 2)$ or poor $(\mathrm{mRS}>2)$. We used stepwise logistic regression to select variables for the final model. ROC curve analysis was done to identify the best cut-off for infarct growth rate.

Results We identified 123 patients met the inclusion criteria. Patients with poor outcome showed significant higher rates of age $>80(35 \%$ vs. $15 \%, \mathrm{p}<0.001)$, female gender $(60 \%$ vs. $46 \%, p=0.024)$, coronary artery disease $(22 \%$ vs. $10 \%$, $\mathrm{p}=0.011)$, atrial fibrillation $(39 \%$ vs. $16 \%, \mathrm{p}<0.001)$, and NIHSS $>18$ (55\% vs. $24 \%, \mathrm{p}<0.001)$ than patients with good outcome. Furthermore, patients with poor outcome had higher Tmax $10 \mathrm{sec}$ (mean $62.2 \mathrm{sec}$ vs. $44.4 \mathrm{sec}, \mathrm{p}=0.021$ ) and infarct growth rate (mean $23.6 \mathrm{ml} / \mathrm{h}$ vs $8.3 \mathrm{ml} / \mathrm{h}, \mathrm{p}=0.007$ ). Whereas, the two groups were similar for the infarct volume before revascularization (mean $19.3 \mathrm{ml}$ vs. $26.6 \mathrm{ml}, \mathrm{p}=0.175$ ). Female sex (OR: 2.92, CI: $1.20-7.49$ ), presence of atrial fibrillation (OR: 2.84, CI: $1.02-8.40$ ), NIHSS $>18$ (OR: 3.44, CI: 1.35 - 9.21) and a higher infarct growth rate (OR:7.17, CI: 2.12 - 35.2) were independently associated with poor functional outcome at 3 months follow-up. Furthermore, the ROC analysis showed an infarct growth rate of 3 $\mathrm{ml} / \mathrm{h}$ (AUC: 0.7 ) with the highest sensitivity (71\%) and specificity (52\%) for distinguishing between the slow and fast progressors.

Conclusions Failure of hemodynamic compensation measured by infarct growth rate represents an important predictor of poor functional outcome independent of recanalization. Early identification of transfer patients with greater infarct growth rates could help select those patients to alternative triaging systems such as direct to OR to minimize infarct progression.

Disclosures D. Quispe Orozco: None. J. Sequeiros Chirinos: None. C. Zevallos Mau: None. A. Mendez Ruiz: None. S. Abdelkarim: None. S. Ansari: None. A. Mendez Ruiz: None. 
M. Farooqui: None. S. Dandapat: None. S. Ortega Gutierrez: 2; C; Medtronic, Stryker.

\section{E-092 EFFECT OF STENT RETRIEVER THROMBECTOMY VS. COMBINED TECHNIQUE ON REVASCULARIZATION IN PATIENTS WITH LARGE VESSEL OCCLUSION STROKE: CASE-CONTROL STUDY}

M Mohammaden*, D Haussen, L Pisani, A Al-Bayati, A Anderson, B Liberato, M Frankel, R Nogueira. Marcus Stroke and Neuroscience Center, Grady Memorial Hospital and Department of Neurology, Emory University School of Medicine, Atlanta, GA

\subsection{6/neurintsurg-2020-SNIS. 125}

Background and Purpose Stent-retriever (SR) thrombectomy remains the best proven treatment modality for anterior circulation large vessel occlusion strokes (LVOS). Three randomized clinical trials have reported similar safety and efficacy for contact aspiration (CA) and SR thrombectomy. The ASTER-2 trial compared combined technique (SR + CA) versus SR alone. The study failed to demonstrate differences in terms of final reperfusion and clinical outcomes across the two modalities. However, reperfusion was significantly better after the allocated treatment only with combined technique. We aim to determine whether the combined technique is superior to SR thrombectomy alone as first line strategy in a large single-center cohort where balloon-guide catheter was universally used.

Methods A prospectively maintained mechanical thrombectomy database from January 2018 to December 2019 was reviewed. Patients were included if they had anterior circulation LVO due to intracranial ICA or MCA-M1 segment and underwent SR thrombectomy or combined technique as first-line therapy. The cohort was divided into two groups matched for age, baseline NIHSS score, prior IV-tPA, site of occlusion, stroke etiology, use of general anesthesia and balloon guide catheter. The primary outcome was the achievement of first-pass effect (FPE) (mTICI2c-3). Secondary outcomes included modified FPE, number of passes, successful reperfusion (mTICI2b-3) without and after rescue techniques and functional independence (90-day mRS 0-2). Safety outcomes included the rates of parenchymal hematoma (PH) type 2 and 90-day mortality. A secondary analysis was performed for the interaction between different subgroups and thrombectomy modality on successful reperfusion.
Results A total of 244 patients (122 pairs) were eligible for the analysis. Patients with first-line combined technique had similar rates of FPE $(56.6 \%$ vs. $51.6, \mathrm{p}=0.44)$, successful reperfusion without $(77 \%$ vs. $77.9 \%, \quad \mathrm{p}=0.88)$ and after (99.2\% vs. $99.2 \%, p=1.00)$ rescue techniques and 90 -day functional independence $(46.6 \%$ vs. $54.4 \%, \mathrm{p}=0.30)$ compared to SR thrombectomy. However, first-line combined technique had a significantly higher number of passes and longer reperfusion times compared to SR alone $(\mathrm{p}=0.005$ and $\mathrm{p}=0.01$, respectively). The rates of $\mathrm{PH}$ type 2 and 90-day mortality were comparable between both groups. There was no significant interaction between age, prior IV-tPA, site of occlusion and presence of favorable collaterals and the first-line treatment modality in terms of successful reperfusion.

Conclusions Our findings reinforce the findings of ASTER-2 in that the first-line thrombectomy with a combined technique did not result in increased rates of successful reperfusion or a better outcome. In contrast with ASTER-2, we did not see any advantage even prior to rescue devices.

Disclosures M. Mohammaden: None. D. Haussen: 2; C; Stryker, Vesalio. 4; C; Viz.AI. L. Pisani: None. A. Al-Bayati: None. A. Anderson: None. B. Liberato: None. M. Frankel: None. R. Nogueira: 2; C; Medtronic, Anaconda, Genentech, Brainomix, Viz-AI, Corindus Vascular Robotics.

\section{E-093 ENDOVASCULAR TREATMENT OF ACUTE CAROTID ATHEROSCLEROTIC TANDEM OCCLUSIONS: PREDICTORS OF CLINICAL OUTCOMES AS TECHNICAL ASPECTS AND LOCATION OF TANDEM OCCLUSIONS}

${ }^{1} \mathrm{E}$ Yoon, ${ }^{2} \mathrm{~S} \mathrm{Ha}^{*} .{ }^{1}$ Radiology, Chosun univ. Hospital, Gwangju Metropolitan City, Korea, Republic of; ${ }^{2}$ Neurosurgery, Chosun univ. Hospital, Gwangju Metropolitan City, Korea, Republic of

\subsection{6/neurintsurg-2020-SNIS. 126}

Objective We aimed to analyze angiographic and clinical outcomes according to the sequence of treatment (antegrade versus retrograde) in patients with acute ischemic stroke caused by tandem extracranial cervical carotid and intracranial large vessel occlusion.

Methods All eligible tandem occlusion patients from April 2012 to March 2019 undergoing carotid artery stenting (CAS) simultaneously with intracranial endovascular thrombectomy (EVT) were retrospectively reviewed. After dividing into 2 